\title{
Microwave-enhanced synthesis of biodegradable multifunctional chitosan hydrogels for wastewater treatment
}

\author{
M. Piątkowski ${ }^{*}$, E. Janus ${ }^{1}$, J. Radwan-Pragłowska ${ }^{1}$, K. Raclavsky ${ }^{2}$ \\ ${ }^{1}$ Department of Biotechnology and Physical Chemistry, Faculty of Chemical Engineering and Technology, Cracow \\ University of Technology, Warszawska 24 St, 31-155 Cracow, Poland \\ ${ }^{2}$ VŠB-Technical University of Ostrava, ENET Centre, 17th November Street, 70833 Ostrava-Poruba, Czech Republic
}

Received 7 February 2017; accepted in revised form 10 May 2017

\begin{abstract}
Chitosan, a derivative of chitin, is a biodegradable polymer known of its favorable properties, applicable in medicine and industry. Commonly obtained chitosan hydrogels are of various swelling capacity, and may bind only anions losing their susceptibility to biodegradation. Hydrogels are mostly obtained using toxic crosslinkers, which pollute environment due to waste generation during their synthesis. In the present article a novel, waste-free method for obtaining chitosan hydrogels under microwave irradiation, is described. Their chemical and morphological structure, swelling properties, sorption capability of a model dye and cadmium ions are described, and kinetic studies, were carried out. Biodegradability of the obtained hydrogels was investigated with the Sturm Test method. As a result, multifunctional chitosan hydrogels with both negative and positive surface charges and increased ability of anions and cations binding, were obtained. Materials were fully biodegradable, capable to absorb high amounts of water, as well as to remove various water contaminants.
\end{abstract}

Keywords: biodegradable polymers, wastewater treatment, microwave synthesis, biodegradation studies, green chemistry

\section{Introduction}

Chitosan is a versatile polysaccharide derivative obtained via chemical or enzymatic chitin deacetylation, which is a main component of crustaceans and mollusks exoskeletons. This biopolymer is characterized by biological activity, biocompatibility or biodegradability and has many different morphological forms, among others hydrogels, and wide application in medicine (e.g. controlled drug release or drug delivery systems) and industry (e.g. water and waste waters treatment). Due to free protonated amino groups, chitosan is defined as a polycation. Therefore, it forms various complexes with compounds possessing negative charge like polymers, proteins and dyes. Moreover, it is capable of selective bonding cholesterol, fats, cancer cells and nucleic acids (deoxyribonucleic acid and ribonucleic acid) [1-5].
Hydrogels are defined as cross-linked hydrophilic polymers having the ability of swelling in water with very high volume increase and can be obtained from monomers by polymerization with the addition of cross-linking agent (e.g. acrylamide polymerization in the presence of $\mathrm{N}, \mathrm{N}$-methylenebisacrylamide) or trough polymers crosslinking. Currently, hydrogels available on the market are mostly non-biodegradable and during synthesis a lot of environmentally harmful substances are used. Therefore, their production is associated with pollution and toxic waste generation, as well as time and money consuming [6-10].

Industrial waste waters are contaminated with heavy metals ions accumulating in living organisms, therefore having toxic influence on them. Some materials of biological origin possessing the ability of adsorbing heavy metals ions can be used for easy removal

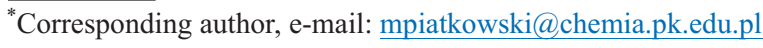
(C) BME-PT 
from the water. Chitosan successfully binds metals ions, both anions and cations via chelating process and/or by electrostatic interactions [11-15]. Physical modification, like mixing with other polymers, and chemical cross-linking or grafted copolymers synthesis make possible biosorbent production characterized by high selectivity and sorption capacity. Therefore, it can be used not only for heavy metals, like mercury or cadmium, but also noble metals (palladium, platinum) or radionuclides (uranium) binding. To extend their application a new procedure must be developed, resulting in preparation of multifunctional sorbent material for the removal of various contaminants [16-20]. In this paper, a novel synthesis method of chitosan hydrogels for waste water treatment is presented. Analysis of physicochemical properties and biodegradation studies, are also discussed. To fulfill Green Chemistry principles, microwave radiation was used for the reduction of time, energy and chemicals consumption. As a result, a novel non-waste synthesis method of fully biodegradable multifunctional hydrogels was developed.

\section{Experimental}

\subsection{Chemical reagents and methods}

All chitosan hydrogels were synthesized using chitosan with a viscosity average molar mass of $410000 \mathrm{~g} / \mathrm{mol}$ and $79 \%$ deacetylation degree purchased from Vanson, Redmond, USA. Bromocresol Green dye, cadmium nitrate, aspartic acid and ethylene glycol were purchased from POCH, Poland. Acetate and phosphate buffers were purchased from Sigma Aldrich, USA.

\subsection{Microwave-assisted chitosan hydrogels synthesis}

For the synthesis of chitosan hydrogels $0.5 \mathrm{~g}$ of biopolymer was dissolved in $2 \%$ acetic acid solution, then $0.1 \mathrm{~g}$ of aspartic acid and $1 \mathrm{~cm}^{3}$ of ethylene glycol, was added. Ready reaction mixture was placed in Prolabo Synthewave 402 microwave reactor. Microwave-assisted chitosan hydrogels synthesis parameters are presented in Table 1.

\subsection{Fourier transform infrared spectroscopy (FT-IR) analysis}

FT-IR analysis was performed using an IR Nicolet 6700 spectrometer, USA. Dried hydrogels samples were embedded into $\mathrm{KBr}$ pellets.
Table 1. Chitosan hydrogels synthesis parameters

\begin{tabular}{|c|c|c|c|}
\hline Sample & $\begin{array}{c}\text { Time } \\
{[\text { min] }}\end{array}$ & $\begin{array}{c}\text { Power } \\
{[\mathbf{W}]}\end{array}$ & $\begin{array}{c}\text { Temperature } \\
{\left[{ }^{\circ} \mathbf{C}\right]}\end{array}$ \\
\hline $\mathrm{a}$ & 20 & 400 & 160 \\
\hline $\mathrm{b}$ & 25 & 400 & 160 \\
\hline $\mathrm{c}$ & 30 & 400 & 160 \\
\hline $\mathrm{d}$ & 20 & 400 & 165 \\
\hline $\mathrm{e}$ & 25 & 400 & 170 \\
\hline $\mathrm{f}$ & 25 & 500 & 165 \\
\hline $\mathrm{g}$ & 30 & 500 & 165 \\
\hline $\mathrm{h}$ & 30 & 500 & 170 \\
\hline $\mathrm{i}$ & 20 & 600 & 170 \\
\hline $\mathrm{j}$ & 25 & 600 & 175 \\
\hline
\end{tabular}

\subsection{Scanning electron microscope (SEM) analysis}

SEM analysis was performed using FEI QUANTA 650 FEG. All samples before the analysis were dried.

\subsection{Swelling properties study}

Swelling properties study was performed by putting dried hydrogel samples into distilled water at room temperature. All hydrogels were weighed before and after swelling.

\subsection{Sorption study}

\subsubsection{Dye sorption}

Dye sorption process was performed at different $\mathrm{pH}$ values (5,7 and 9) to simulate various types of waste water. Solutions were prepared using distilled water and acetate and phosphate buffers. $0.5 \mathrm{~g}$ of chitosan hydrogel was placed in $100 \mathrm{~mL}$ of dye solution. The concentration of bromocresol green was $1000 \mathrm{mg} / \mathrm{L}$. Sorption study was performed under dynamic conditions with an agitation speed of $200 \mathrm{rpm}$. For the $\mathrm{pH}$ measurements, $\mathrm{pH}$-sensitive electrode Hydromet ERH-11 (Poland) was used.

\subsubsection{Heavy metal ions sorption}

Cadmium ions sorption process was investigated at $\mathrm{pH}=5$ to simulate the acidity of the natural waters and waste waters. $0.5 \mathrm{~g}$ of chitosan hydrogel was placed in $100 \mathrm{~mL}$ of dye solution. Sorption study was performed under dynamic conditions with an agitation speed of $200 \mathrm{rpm}$. The concentration of bromocresol green was $1000 \mathrm{mg} / \mathrm{L} . \mathrm{Cd}^{2+}$ ions concentration was measured using BAS 100B Electrochemical Analyzer USA (Differential Pulse Voltammetry method). 


\subsection{Sorption kinetics study}

\subsubsection{Pseudo first-order kinetics}

Sorption kinetics was calculated according to the following mathematic formulas (Equations (1)-(4)):

$$
\frac{\mathrm{d} q_{\mathrm{t}}}{\mathrm{d} t}=k_{1}\left(q_{\mathrm{e}}-q_{\mathrm{t}}\right)
$$

where $q_{\mathrm{e}}$ amount of adsorbed by hydrogel substance in equilibrium state $[\mathrm{mg} / \mathrm{g}], q_{\mathrm{t}}$ amount of adsorbed substance in any time $[\mathrm{mg} / \mathrm{g}], k_{1}$ pseudo first-order rate constant $[1 / \mathrm{min}]$ or $[1 / \mathrm{h}]$.

$$
\begin{aligned}
& \int_{0}^{q_{\mathrm{t}}} \frac{\mathrm{d} q}{q_{\mathrm{e}}-q_{\mathrm{t}}}=\int_{0}^{t} k_{\mathrm{l}} \mathrm{d} t \\
& \ln \frac{q_{\mathrm{e}}-q_{\mathrm{t}}}{q_{\mathrm{e}}}=-k_{1} t \\
& \log \left(q_{\mathrm{e}}-q_{\mathrm{t}}\right)=\log q_{\mathrm{e}}-\frac{k_{1} t}{2.303}
\end{aligned}
$$

\subsubsection{Pseudo second-order kinetics}

Sorption kinetics was calculated according to the following mathematic formulas (Equations (5)-(8)):

$\frac{\mathrm{d} q}{\mathrm{~d} t}=k_{2}\left(q_{\mathrm{e}}-q_{\mathrm{t}}\right)^{2}$

$k_{2}$ - pseudo second-order rate constant $[\mathrm{g} /(\mathrm{mg} \cdot \mathrm{min})]$

$$
\int_{q_{\mathrm{t}}=0}^{q_{\mathrm{t}}=q_{\mathrm{t}}} \frac{\mathrm{d} q}{\left(q_{\mathrm{e}}-q_{\mathrm{t}}\right)^{2}}=\int_{0}^{t} k_{2} \mathrm{~d} t
$$

$\frac{t}{q_{\mathrm{t}}}=\frac{1}{q_{\mathrm{e}}} t+\frac{1}{h_{0}}$

h0 - initial adsorption rate

$h_{0}=k_{2} q_{\mathrm{e}}^{2}$

\subsubsection{Intraparticle diffusivity model}

The linear driving force concept was used to develop the relationship for particle-diffusion controlled adsorption as shown by Equations (9) and (10):

$\ln (1-\alpha)=-K_{\mathrm{p}} t$

where $K_{\mathrm{p}}$ the rate coefficient for the particle-diffusion controlled process corresponding to the particle size of the adsorbent, $\ln (1-\alpha)$ a measure of the intraparticulate diffusivity.

$\alpha=\frac{c_{\mathrm{t}}}{c_{\infty}}$ where $\alpha$ the fractional attainment to equilibrium, $c_{\mathrm{t}}$ the concentration of the adsorbed substance at time $t, c_{\infty}$ the concentration at equilibrium.

\subsection{Biodegradation study}

Biodegradation study was performed by Sturm Test method according to OECD 301B norm. TOC (Total Organic Carbon) of the studied samples was measured using TOC analyzer. Degree of biodegradation was calculated based on the amount of secreted $\mathrm{CO}_{2}$ as the result of the materials degradation by microorganisms in the activated sludge. All tests were performed using flasks filled with distilled water, mineral medium, air purified from carbon dioxide and tested samples of known weight and TOC content. As a control sample sodium acetate was used. Vessels were connected with scrubbers filled with $\mathrm{Ba}(\mathrm{OH})_{2}$ solution. During the biodegradation process secreted $\mathrm{CO}_{2}$ was adsorbed by barium hydroxide which was further used for $\mathrm{CO}_{2}$ mass calculation (Equation (11)):

$\mathrm{BD}=\frac{m_{\mathrm{CO}_{2} \text { ads. }}}{m_{\mathrm{CO}_{2} \mathrm{TOC}}} \cdot 100 \%$

where $\mathrm{BD}$ biodegradation degree, $m_{\mathrm{CO}_{2}}$ ads. amount of secreted $\mathrm{CO}_{2}$ as the result of material biodegradation by activated sludge, $m_{\mathrm{CO}_{2} \text { TOC theoretical }}$ amount of $\mathrm{CO}_{2}$ present in tested samples

\section{Results and discussion}

\subsection{Microwave-assisted chitosan hydrogels synthesis}

All chitosan hydrogels were prepared by crosslinking reaction resulting in 3D structure formation. Best results were observed for samples $a, b, c$ and $d$. Samples $e$ and $f$ were partially burned or did not undergo crosslinking process. In case of samples $g-j$ complete thermal degradation has occurred due to the harsh synthesis conditions. Therefore the following studies were performed for the hydrogels with the best properties.

As a result of the synthesis, light-brown jelly-like products were obtained as presented in Figure 1. Depending on the synthesis parameters hydrogels of various crosslinking degrees were prepared, what affected sorption properties. To evaluate the influence of microwave radiation on the crosslinking process an alternative synthesis using conventional heating, was performed. In the traditional method no functional hydrogels were obtained. Application of microwave radiation not only enabled crosslinking process using 

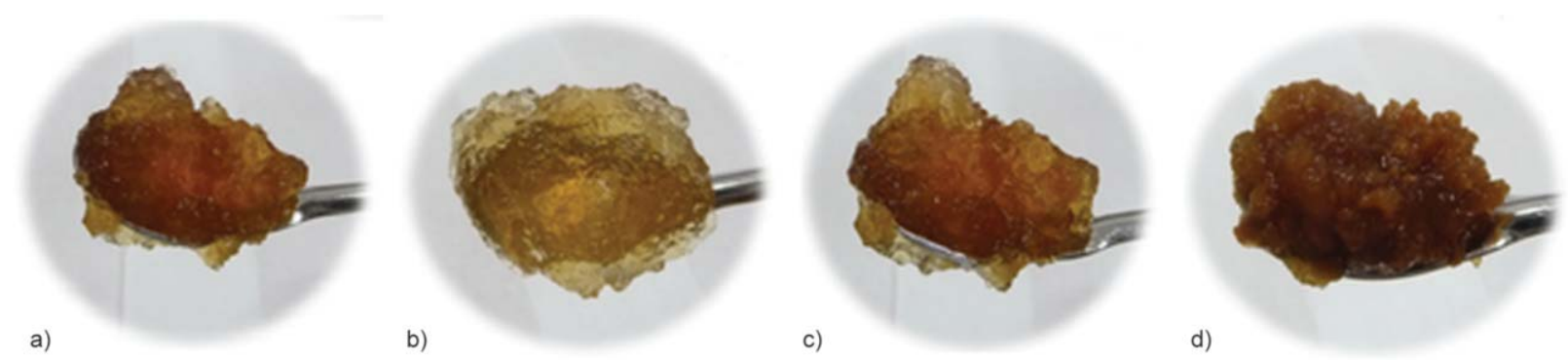

Figure 1. Chitosan hydrogels obtained according to data presented in Table 1 (sample $a, b, c$ and $d$ )

non-toxic and ecofriendly crosslinkers, but also modified the polymer surface. Chitosan surface charge is positive due to free $\mathrm{NH}_{2}$ groups presence. Application of microwave radiation led to the oxidation of hydroxymethyl groups resulting in the formation of carboxyl groups responsible for negative surface charge of the hydrogels in basic environment, thus extending their applicability. Negative charge makes possible the binding of various cations of heavy metals and different toxic substances, what is hard or impossible to achieve without chemical modifications. Such modification enables also various cells attachment improving material susceptibility to biodegradation.

\subsection{FT-IR analysis}

In the spectrum of the obtained hydrogel presented in the Figure 2 typical bands for chitosan are observed: wide band in the range of $3700-3300 \mathrm{~cm}^{-1}$ corresponding to stretching vibrations of hydroxyl and amine groups; symmetric and asymmetric stretching vibrations of methylene groups at 2926.65, $1387.30 \mathrm{~cm}^{-1}$ corresponding to the ethylene glycol and chitosan pyranose ring; stretching vibrations at $1636.24 \mathrm{~cm}^{-1}$ typical for $\mathrm{C}=\mathrm{O}$ (amide groups) and deformation vibrations typical for $\mathrm{N}-\mathrm{H}$ groups; $\mathrm{C}-\mathrm{H}$

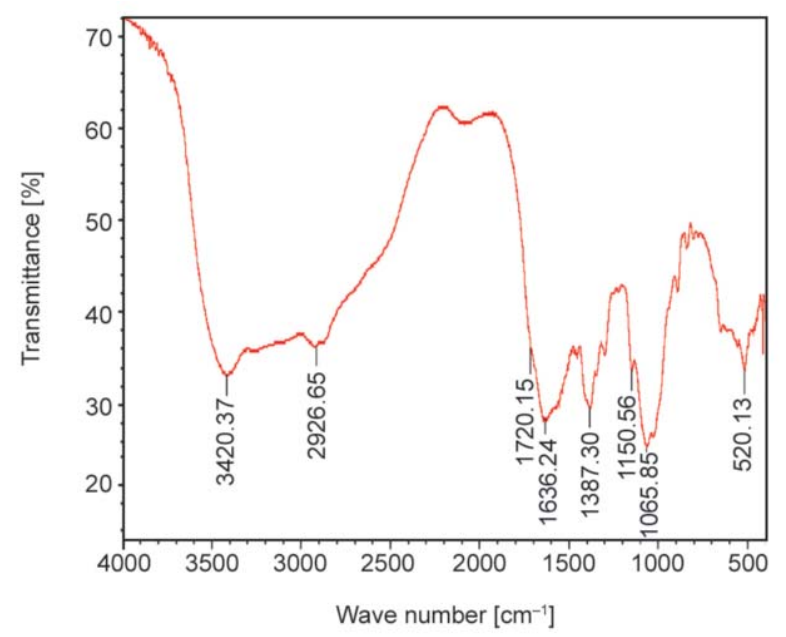

Figure 2. FT-IR spectrum of the chitosan hydrogel (sample $b$ ) deformation vibrations in methyl group in $\mathrm{N}$-acetyl moiety at $1387.30 \mathrm{~cm}^{-1} ;-\mathrm{C}-\mathrm{O}-\mathrm{C}-$ stretching vibrations in glycosidic bond with the maximum at $1065.85 \mathrm{~cm}^{-1}$. Moreover, at $1720.15 \mathrm{~cm}^{-1}$ a band from free carboxylic groups formed due to the biopolymer degradation under microwaves irradiation, can be noticed.

The FT-IR analysis confirmed three dimensional structure of the hydrogel (Figure 3). Chitosan macromolecules are cured with esters of aspartic acid and ethylene glycol grafted to biopolymer with ester and amide linkages. Free amino groups are more reactive than hydroxyl ones in chitosan, therefore in the first step of condensation, a reaction of amino groups of biopolymer with carboxyl groups of aspartic acid, is observed. Afterwards, with water evaporation creation of two types of esters occurred, resulting in gel formation. Local overheating due to microwaves caused self-condensation of aspartic acid, what additionally crosslinked the structure. Absorption of water results in chitosan-based hydrogel formation. Moreover, no unreacted aspartic acid was found in the final product what confirms full incorporation into polymer.

\subsection{SEM analysis}

SEM microphotographs (Figure 4) demonstrate crosslinked chitosan hydrogels. A very porous structure, typical for hydrogels, can be observed. Pore sizes are larger than microorganisms cells diameter, leading to biofilm formation on its surface, and to the penetration of enzymes and metabolites through the crosslinked structure, accelerating the biodegradation process. A correlation between synthesis conditions and hydrogels' morphology can be observed. In case of samples $a$ the pores of bigger size and more regular shape are due to the lower temperature and irradiation time during the synthesis. It can be caused by the lower crosslinking degree resulting in less branched structure which is associated with a lower number of bonds between the polymer and crosslinking agents. 


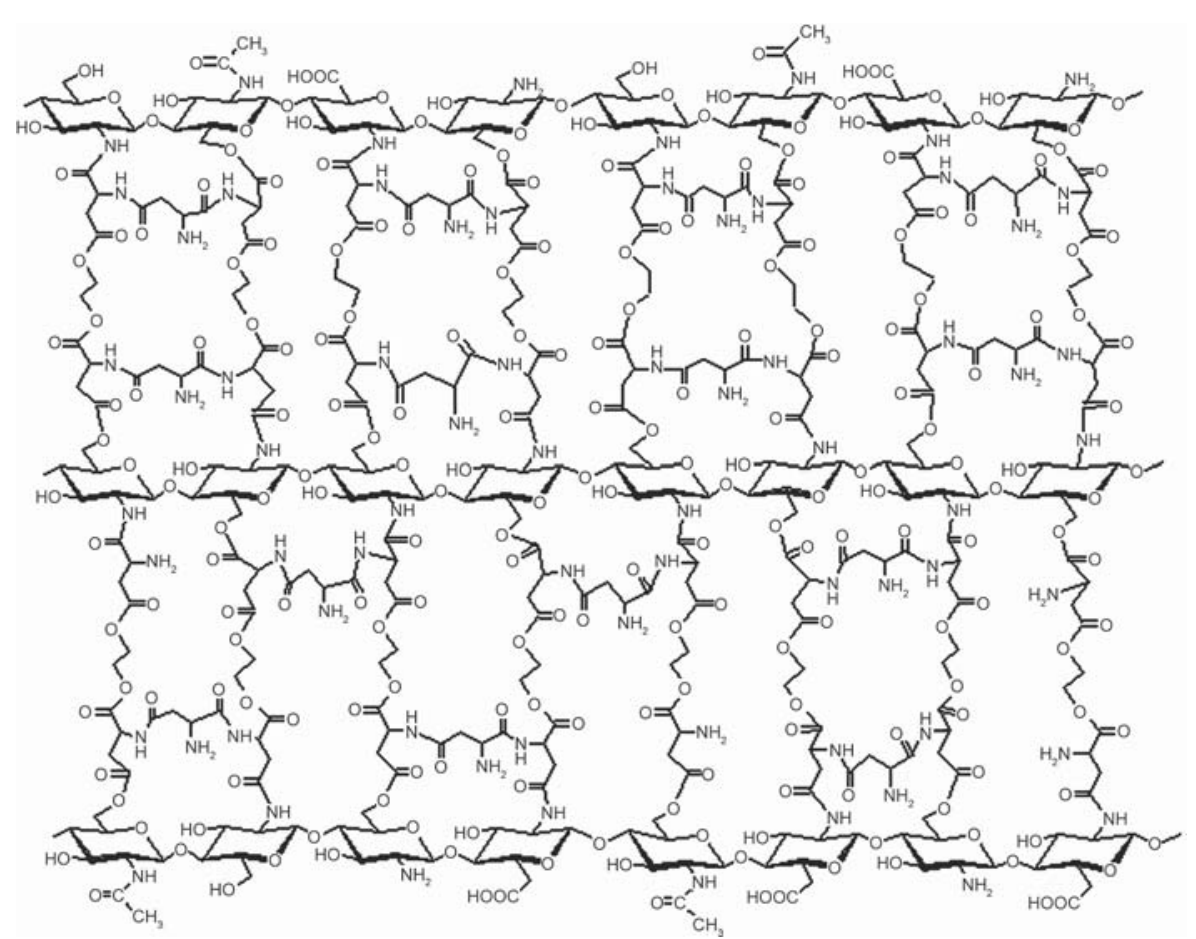

Figure 3. Proposed chemical structure of the obtained hydrogels in the crosslinking reaction between chitosan, ethylene glycol and aspartic acid

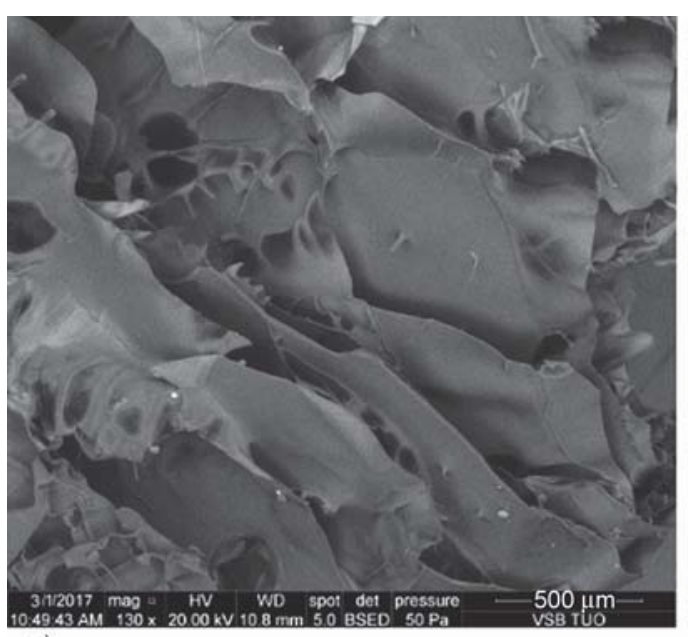

a)

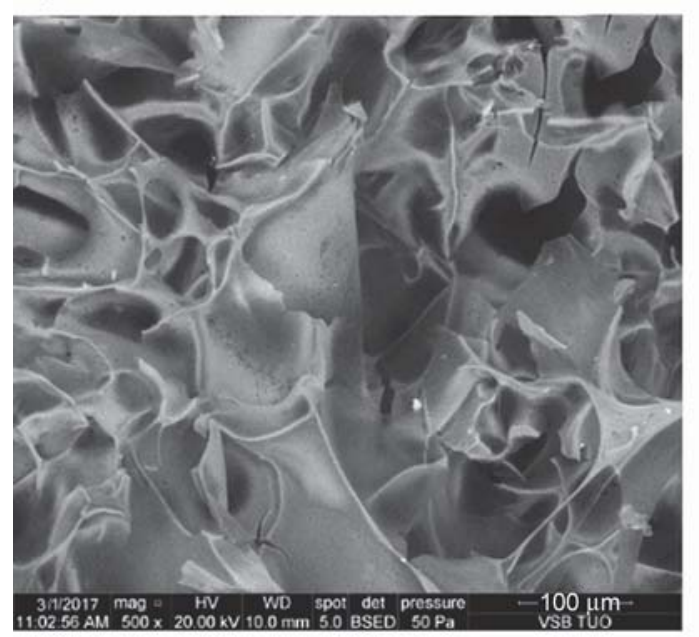

c)

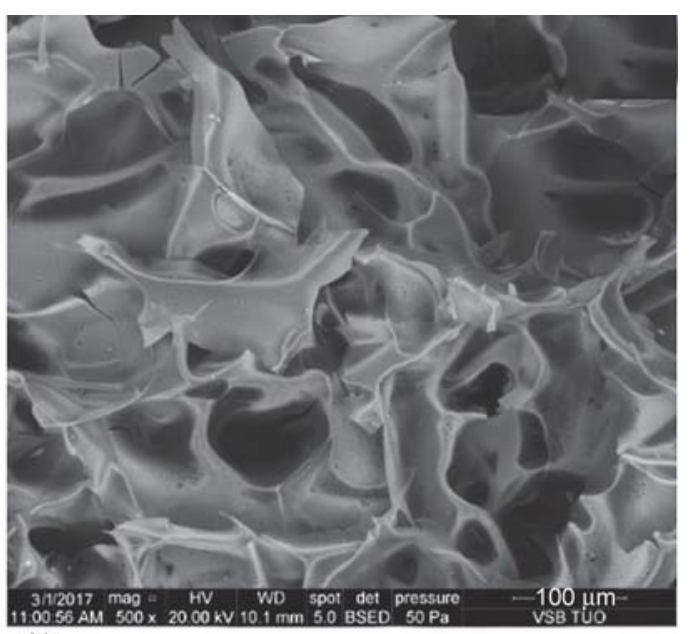

b)

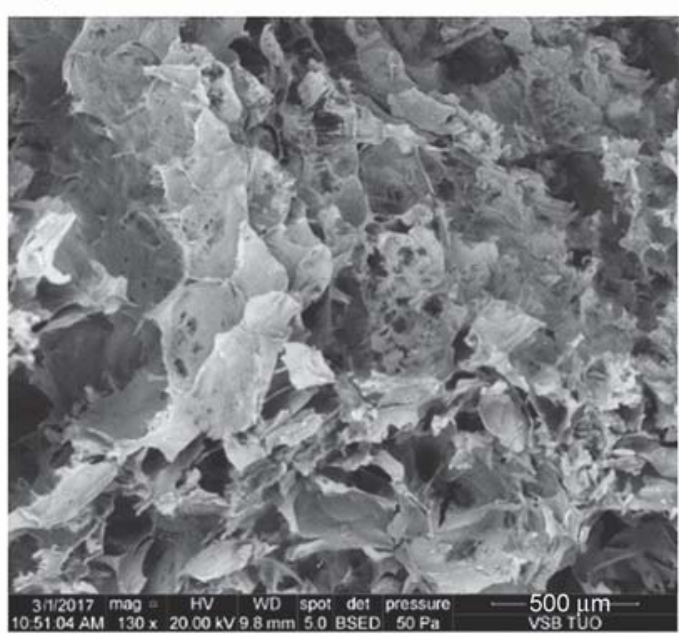

d)

Figure 4. SEM microphotographs of samples $a, b, c$ and $d$ obtained according to data presented in Table 1 
Samples $b$ and $c$ exhibit similar porosity. That the size and shape of pores are less regular than in the case of sample a is a consequence of the longer irradiation time. In the case of sample $d$ the pore structure is significantly different from that of other samples. More dense morphological structure can be a result of the higher crosslinking degree which is a result of higher temperature applied during the synthesis. Moreover slight cracks can be noticed connected with the thermal decomposition of the sample. Higher porosity and smaller pores resulted in lower biodegradability and significantly worse swelling capability in comparison with other samples. Pours sizes generate possibility for an efficient adsorption of metal ions, organic particulates and other water pollutants. Additionally, this type of structure enables adhesion of various microorganisms as well as their penetration into the hydrogel matrix, thus providing excellent conditions for biofilm formation resulting in good biodegradation performance. Moreover, this type of morphology creates potentiality for further modifications of the hydrogels, like preparation of in-situ nanoparticles, nanowires, nanotubes or other morphological forms.

\subsection{Swelling properties study}

Best swelling properties illustrated in the Figure 5 were obtained for samples $a, b, c$ and $d$. The highest swelling degrees were observed in the case of sample $b$, which was could absorb $125 \mathrm{~g}$ of water per $1 \mathrm{~g}$ of the hydrogel. The amount of the absorbed water is highly correlated with the number of hydroxyl groups present in the hydrogel structure, as well as its crosslinking degree. Since the highest reactivity was observed between $\mathrm{NH}_{2}$ and $\mathrm{COOH}$ groups resulting in

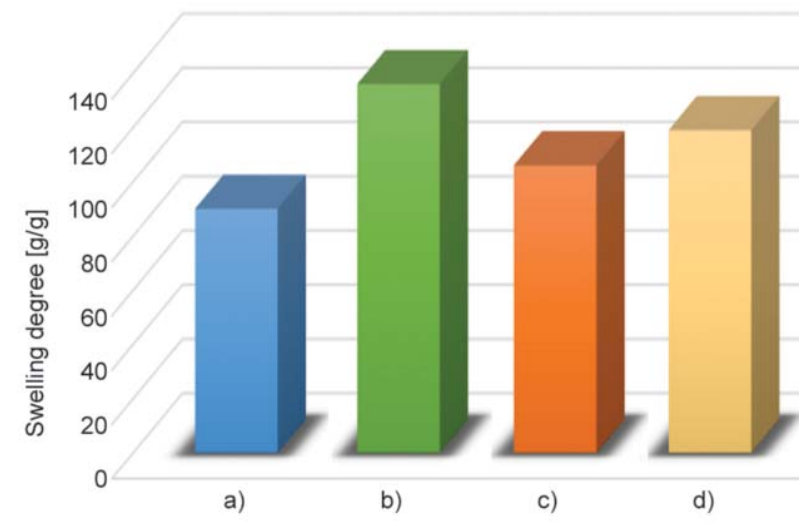

Figure 5. Swelling degree of chitosan hydrogels obtained according to data presented in Table 1

amide bond formation, it can be assumed that most of the $\mathrm{OH}$ groups were unmodified. Hydroxyl groups are responsible for the formation of hydrogen bonds with water molecules. Branched structure of the obtained polymers rich in $\mathrm{OH}$ groups coming from chitosan pyranose rings enables migration and stabilization of the water inside the 3D structure.

\subsection{Sorption study}

\subsubsection{Dye sorption kinetics study}

Obtained hydrogels were characterized by very good dye sorption capacity (Figure 6). Tests performed at various $\mathrm{pH}$ values confirmed the presence of both negative and positive charges on the hydrogel surface. The best results of sorption study of negatively charged dye were observed at neutral $\mathrm{pH}$ value. Under such conditions a hydrogel was capable to adsorb $156 \mathrm{mg}$ of dye per $1 \mathrm{~g}$ of material. High sorption capacity of the hydrogel can be caused by three-dimensional, porous structure of the polymer enabling easy dye diffusion. Bromocresol Green molecules despite
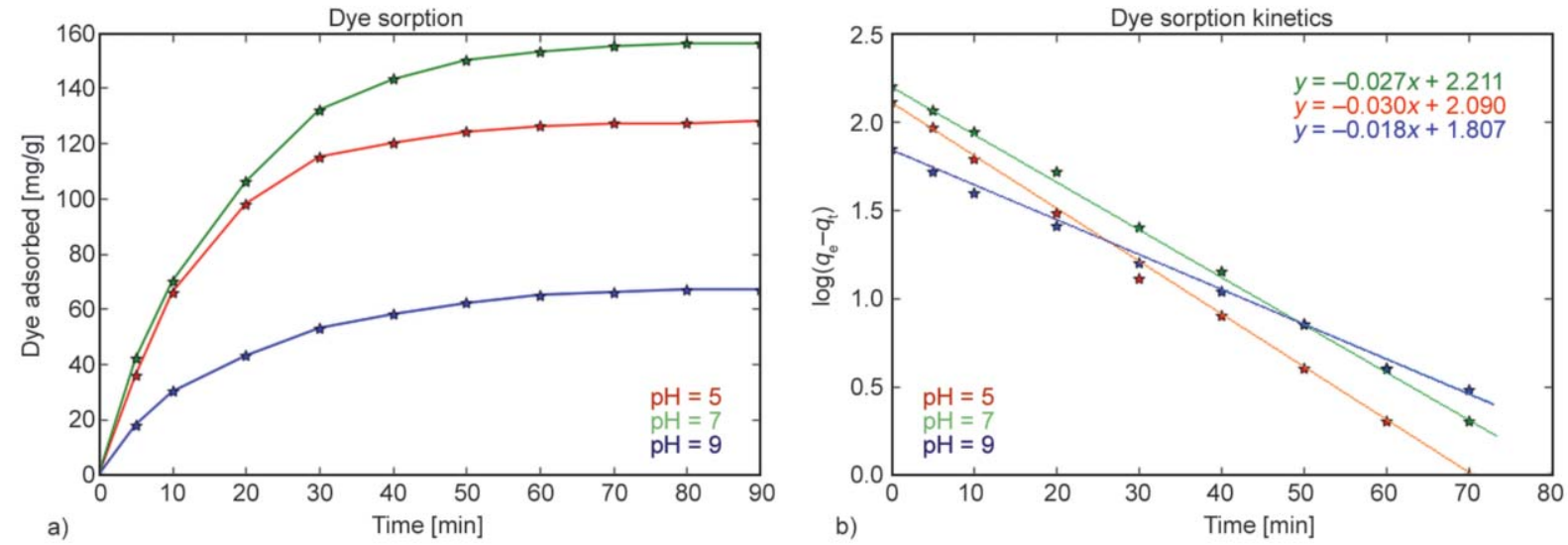

Figure 6. Dye sorption (a) performed in aquatic solutions of $\mathrm{pH}=5,7$ and 9 of the chitosan hydrogel (sample $b$ ) and pseudo first-order kinetics model of dye sorption (b) 
their complexed structure and size were able to penetrate into polymeric matrix due to an appropriate crosslinking degree. It can be noticed that lack of the surface charge of the hydrogel and not dissociated sulphone groups of the dye molecules had a positive effect on Bromocresol Green. Lack of attractions between $\mathrm{SO}_{3}^{-}$and $\mathrm{NH}_{3}^{+}$enabled diffusion of the dye molecules into the three-dimensional structure of the hydrogels and pores of the hydrogels remained unblocked what could happen in case of samples in solutions with different $\mathrm{pH}$ value due to the interactions of the positively and negatively charged groups. Thus it can be assumed that very good dye sorption capacity of the chitosan hydrogels is mainly caused my their morphology, not the presence of free amine groups left unbonded after crosslinking process which are responsible for negative surface charge in acidic $\mathrm{pH}$. Very good results were obtained also in case of $\mathrm{pH}=5$. For $1 \mathrm{~g}$ of the hydrogel adsorption of $137 \mathrm{mg}$ of the dye, was observed. Proposed dye in acidic $\mathrm{pH}$ exists in anion form, therefore interaction with protonated $\mathrm{NH}_{2}$ groups present at the surface of the chitosan polymer, was possible. Formation of electrostatic interactions between negative charge of the Bromocresol Green and positive charge at the hydrogel surface enhanced dye bonding, but only on the top layer of the chitosan material. Attracted dye molecules blocked pores of the hydrogels, therefore impeding dye particles penetration inside the polymeric matrix. The worst sorption results were noticed at basic $\mathrm{pH}=9$. Sorption capacity was significantly lower than in case of neutral and acidic environment (only $57 \mathrm{mg} / \mathrm{g}$ ). Such results may be caused by the fact that negatively charged dye was repulsed by carboxyl groups presented at the hydrogel surface. Such

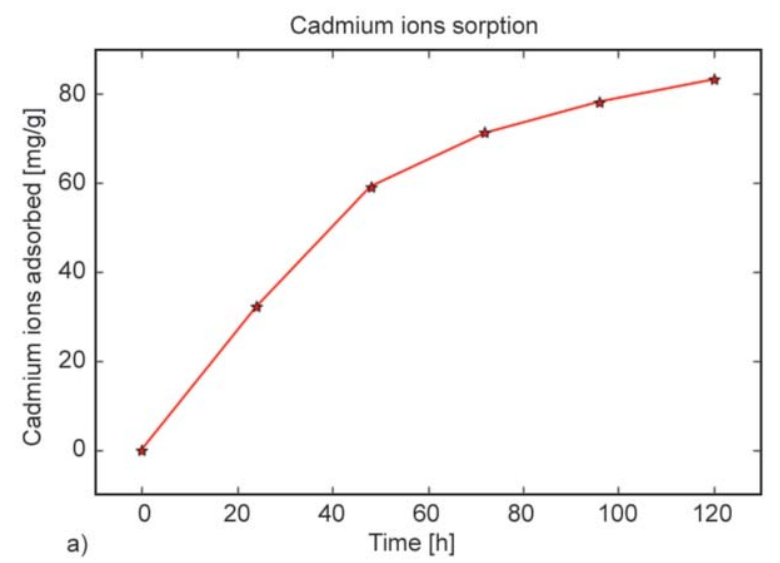

behavior prevented dye molecules from penetrating pores of the polymeric matrix.

For complex characterization of dye sorption process two different kinetics models were analyzed (pseudo first-order and pseudo second-order). Also intraparticle diffusivity model was investigated. Mathematical model characterizing Bromocresol Green sorption process was of pseudo first-order in case of $\mathrm{pH}=5$ and 7 (correlation coefficients of pseudo first-order model are $R^{2}=0.9984$ and 0.9992 and for pseudo second-order $R^{2}=0.9943$ and 0.9948$)$. In case of $\mathrm{pH}=9$ sorption process was best described by pseudo second-order model (correlation coefficient of pseudo first-order model is $R^{2}=0.9976$ and for pseudo second-order $R^{2}=0.9983$ Figure 8). Appriopriate kinetic models were chosen basing on the highest correlation coefficient value. Speed rate constant for hydrogel dye sorption in 5 and $7 \mathrm{pH}$ value were $1.30 \cdot 10^{-2}$, $1.17 \cdot 10^{-2}[1 / \mathrm{min}]$ and $1.09 \cdot 10^{-3}[\mathrm{mg} / \mathrm{g} \mathrm{min}]$ for $\mathrm{pH}=9$. Several factors can control adsorption rate: the diffusion of dissolved substances from the solution to the film surrounding the particle, external diffusion or surface/ poor diffusion. The uptake may involve a few mechanisms including: ion exchange, physicochemical sorption, complexation or precipitation. Thus the rate of attainment to equilibrium can be film or particle-diffusion controlled. The correlation coefficients for Interparticle Diffusivity Model for dye sorption in case of $\mathrm{pH}=5,7$ and 9 are: $R^{2}=$ $0.9955,0.9468$ and 0.9429 respectively. When $\ln (1-\alpha)$ versus time plot is linear the adsorption process is described as particle-diffusion controlled. In such case diffusivity of Bromocresol Green onto the hydrogel surface is independent of the extent of adsorption. The Figure 9 demonstrates correlation

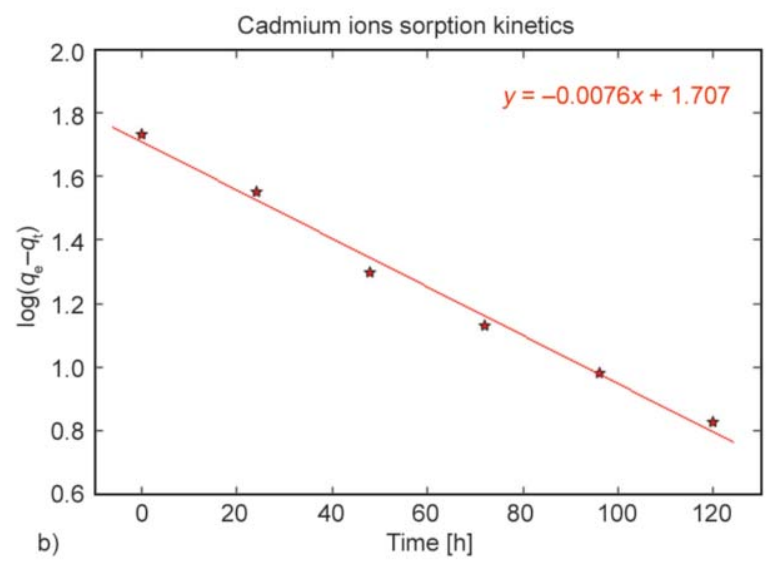

Figure 7. Cadmium ions sorption (a) performed in aquatic solution of $\mathrm{pH}=5$ of the chitosan hydrogel (sample $b$ ) and pseudo first-order kinetics model of cadmium ions sorption (b) 

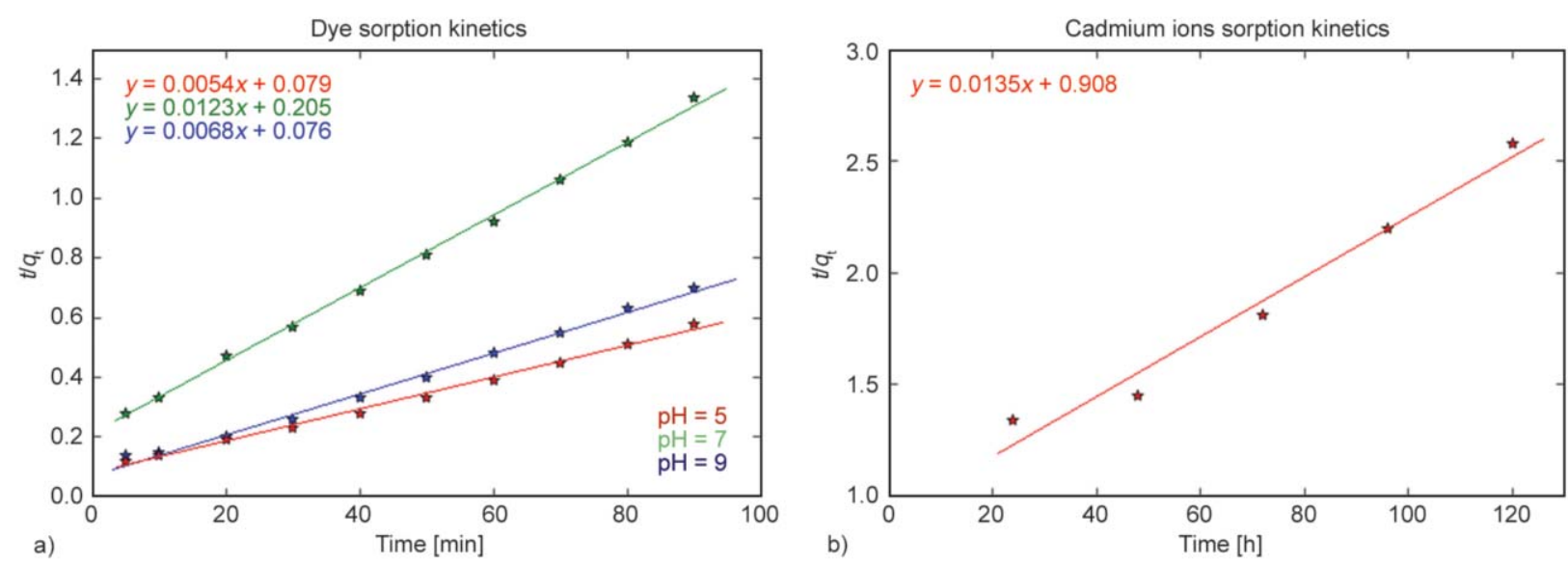

Figure 8. Pseudo second-order kinetics model of dye sorption (a) performed in aquatic solutions of $\mathrm{pH}=5,7$ and 9 of the chitosan hydrogel (sample $b$ ) and pseudo second-order kinetics model of cadmium ions sorption (b) performed in aquatic solution of $\mathrm{pH}=5$ of the chitosan hydrogel (sample $b$ )

between $\ln (1-\alpha)$ and time which is linear only in case of adsorption in $\mathrm{pH}=5$. Therefore adsorption in neutral and basic $\mathrm{pH}$ cannot be described as particle diffusion model.

\subsubsection{Heavy metal ions sorption kinetics study} Chitosan hydrogels maintained ability of ions binding (Figure 7). Three dimensional polymeric structure was capable of ions complexing due to the presence of hydroxyl and amino groups. Functional groups $\left(\mathrm{OH}\right.$ and $\mathrm{NH}_{2}$ ) were able to bind $\mathrm{Cd}^{2+}$ ions through their coordination and immobilization inside hydrogel matrix. Obtained materials were able to adsorb $80 \mathrm{mg}$ per $1 \mathrm{~g}$. Proposed chitosan hydrogels have equal or better ion sorption capacity in comparison to other advanced sorbents. Such materials are time consuming to obtain and are not biodegradable. Moreover many of them have complicated preparation methods using toxic and corrosive reagents. The degradation of some sorbents may lead to the formation of substances harmful for aquatic ecosystem [21-23]. Heavy metal ions adsorption process in comparison to dye sorption is more time-consuming and complicated. Positive charge of the metal ions could cause their repulsions by protonated amino groups. Bonded cadmium ions after their adsorption on the hydrogels (water treatment) could be removed from the materials.

Mathematical model describing cadmium ions adsorption process is analogical to the dye sorption kinetics. $\mathrm{Cd}^{2+}$ ions sorption occurred as pseudo firstorder kinetics model (correlation coefficient for first-order $R^{2}=0.9976$ and for second-order $R^{2}=$ 0.9722 ). Equilibrium state was achieved after two days. After 50 hours speed rate has decreased what could be caused by the reduced number of free hy-
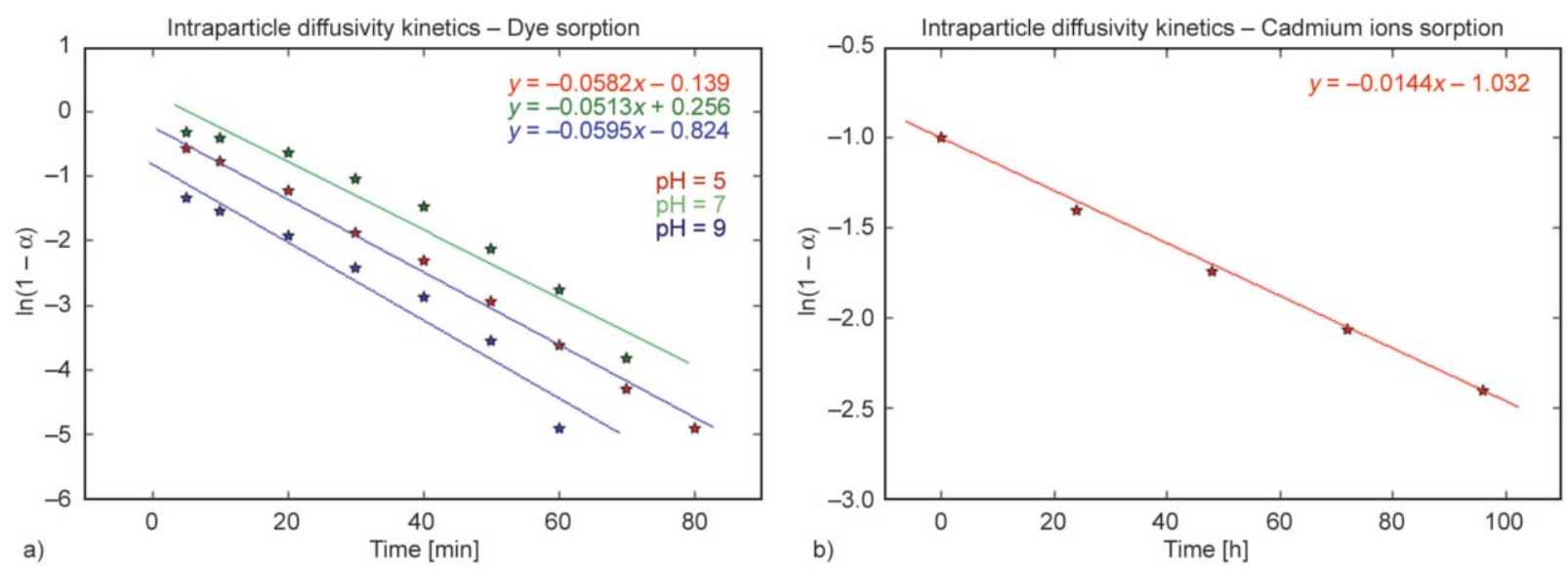

Figure 9. Interparticle diffusivity kinetics model of dye sorption (a) performed in aquatic solutions of $\mathrm{pH}=5,7$ and 9 of the chitosan hydrogel (sample $b$ ) and interparticle diffusivity kinetics model of cadmium ions sorption (b) performed in aquatic solution of $\mathrm{pH}=5$ of the chitosan hydrogel (sample $b$ ) 
droxyl and amino groups capable of ions binding. High speed rate at the beginning of the process can be associated by the number of $\mathrm{OH}$ and $\mathrm{NH}_{2}$ groups on the surface of the material. Further adsorption progress was limited since cadmium ions had to diffuse into the 3D structure of the hydrogel, where the amount of free hydroxyl and amino groups was much lower due to amide and ester bonds formation as the result of crosslinking process. According to the kinetics model speed rate of the process was 0.018 [1/hour]. Basing on the value of correlation coefficient $R^{2}=0.9981$ it can be stated that adsorption process of cadmium ions on the surface of chitosan hydrogel is particle-diffusion controlled.

\subsection{Biodegradation study}

As presented in the Figure 10, all hydrogels biodegraded in at least $60 \%$ during 28 days, therefore they can be considered as biodegradable materials. There are no significant differences between samples $a$ and $b$ due to the small change in crosslinking degree of the hydrogels. Sample $b$ was biodegraded in approximately $90 \%$. Hydrogels which were of higher cross-linkage (samples $c$ and $d$ ) were less biodegradable due to the more branched and thick structure, which impeded microorganism adhesion and migration into polymer $3 \mathrm{D}$ structure. It can be also connected with the higher amount of bondings necessary to hydrolyze by bacterial enzymes. Due to similarities in chemical structure to natural polymers like cellulose or chitin, there was no need to apply special microorganisms species, like in case of biodegradation of e.g. polyacrylamides. The biodegradation process can be divided into three phases: adaptation, biodegradation and stationary phase.

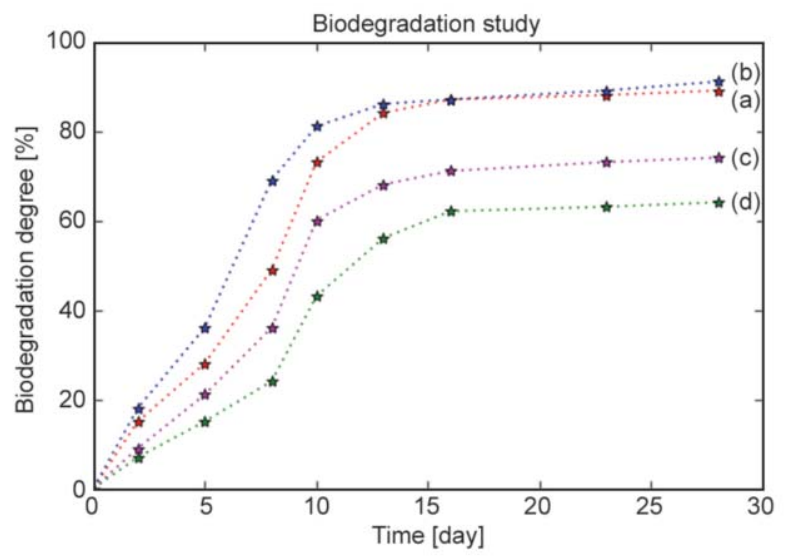

Figure 10. Biodegradation study of the obtained hydrogels according to OECD 301B norm for samples $a, b$, $c$ and $d$
In the first phase microorganisms present in the activated sludge attached to the hydrogels surface, what was possible due to their hydrophilic character, as well as carboxylic groups presence. Although, crosslinked polymers are considered as difficult in biodegradation, high porosity of the obtained materials enabled microorganisms adhesion and migration into three-dimensional structure.

In the second phase the main biodegradation process has occurred. Due to the presence of microorganisms, which can secrete some enzymes and metabolites, like strong organic acids, it may be assumed that the biocorrosion preceded biological degradation. In this process some covalent bonds were broken enabling further penetration into the polymeric structure due to the deterioration of mechanical properties of the material. It can be assumed that degradation process has started with breaking glycosidic bonds by endoend exoenzymes resulting in biofragmentation. Significantly developed surface and high porosity probably enabled further penetration of enzymes into the polymeric matrix resulting in enzymatic hydrolysis or oxidation of the bonds.

Last stationary phase can be described as biomineralization process, which is very slow and the amount of $\mathrm{CO}_{2}$ secreted is low. In this process mineral compounds secreted by microorganisms are non-toxic to the environment, since they are incorporated into geochemical cycles.

The results of the performed measurements indicate that hydrogel obtained via microwave synthesis is characterized by the highest sorption capacity values in neutral $\mathrm{pH}$ due to its development of large specific surface area. Obtained material is highly crosslinked and rich in oxygen and nitrogen atoms. Therefore, multiple hydrogen bonds may form what enables dye sorption. Moreover, functional groups present at the surface $\left(-\mathrm{COOH},-\mathrm{NH}_{2}\right.$ and $\left.-\mathrm{OH}\right)$ may be also involved into adsorption process. As a result, obtained material has very high potential for its application as an efficient adsorbent for organic compounds elimination from waste water and sewage.

\section{Conclusions}

In this article novel environment-friendly chitosan hydrogels obtainment method is presented. Application of appropriate so-called green crosslinkers (ethylene glycol and aspartic acid) as well as microwave irradiation enabled preparation of the materials without generation of any wastes. Ready hydrogels had 
both negatively charged $\left(\mathrm{COO}^{-}-\right.$result of chitosan degradation and oxidation under microwave irradiation) and positively charged $\left(\mathrm{NH}_{3}^{+}\right)$functional groups on their surface, thus enhancing sorption properties. Three-dimensional structure was obtained due to polymer branching as a result of ester and amide bonds formation. High porosity as well as number of hydrophilic groups leaded to excellent swelling properties and ability of various water contaminants removal. Obtained materials were fully biodegradable due to the heteroatoms presence in the polymeric matrix, hydrophilic character of the hydrogels, as well as 3D structure enabling enzymes penetration and microorganisms attachment. Proposed materials give an interesting alternative to commonly used hydrogels due to their ecofriendly character, as well as increased functionality in various environments. Therefore, presented method enables chitosan hydrogels with increased applicability in waste water treatment synthesis with no contamination of the environment by used materials. Obtained chitosan hydrogels have also high potential for further modifications due to the presence of reactive groups on its surface and various pores, which generates possibility for the nanoparticles in-situ preparation, what will be the subject of the future study.

\section{References}

[1] Belalia R., Grelier S., Benaissa M., Coma V.: New bioactive biomaterials based on quaternized chitosan. Journal of Agricultural and Food Chemistry, 58, 1582-1588 (2008). https://doi.org/10.1021/jf071717+

[2] Kasaai M. R.: Various methods for determination of the degree of $N$-acetylation of chitin and chitosan: A review. Journal of Agricultural and Food Chemistry, 57, 1667-1676 (2009). https://doi.org/10.1021/jf803001m

[3] Yang R., Li H., Huang M., Yang H., Li A.: A review on chitosan-based flocculants and their applications in water treatment. Water Research, 95, 59-89 (2016). https://doi.org/10.1016/j.watres.2016.02.068

[4] Cheung R. C. F., Ng T. B., Wong J. H., Chan W. Y.: Chitosan: An update on potential biomedical and pharmaceutical applications. Marine Drugs, 13, 5156-5186 (2015).

https://doi.org/10.3390/md13085156

[5] LogithKumar R., KeshavNarayan A., Dhivya S., Chawla A., Saravanan S., Selvamurugan N.: A review of chitosan and its derivatives in bone tissue engineering. Carbohydrate Polymers, 151, 172-188 (2016).

https://doi.org/10.1016/j.carbpol.2016.05.049
[6] Rafique A., Zia K. M., Zuber M., Tabasum S., Rehman S.: Chitosan functionalized poly(vinyl alcohol) for prospects biomedical and industrial applications: A review. International Journal of Biological Macromolecules, 87, 141-154 (2016).

https://doi.org/10.1016/j.ijbiomac.2016.02.035

[7] Rinaudo M.: Chitin and chitosan: Properties and applications. Progress of Polymer Science, 31, 603-632 (2006).

https://doi.org/10.1016/j.progpolymsci.2006.06.001

[8] Olivera S., Muralidhara H. B., Venkatesh K., Guna V. K., Gopalakrishna K., Kumar Y. K.: Potential applications of cellulose and chitosan nanoparticles/composites in wastewater treatment: A review. Carbohydrate Polymers, 153, 600-618 (2016).

https://doi.org/10.1016/j.carbpol.2016.08.017

[9] Hamed I., Özogul F., Regenstein J. M.: Industrial applications of crustacean by-products (chitin, chitosan, and chitooligosaccharides): A review. Trends in Food Science and Technology, 48, 40-50 (2016).

https://doi.org/10.1016/j.tifs.2015.11.007

[10] Vakili M., Rafatullah M., Salamatinia B., Abdullah A. Z., Ibrahim M. H., Tan K. B., Gholami Z., Amouzgar P.: Application of chitosan and its derivatives as adsorbents for dye removal from water and wastewater: A review. Carbohydrate Polymers, 113, 115-130 (2014). https://doi.org/10.1016/j.carbpol.2014.07.007

[11] Wang J., Chen C.: Chitosan-based biosorbents: Modification and application for biosorption of heavy metals and radionuclides. Bioresource Technology, 160, 129141 (2014). https://doi.org/10.1016/j.biortech.2013.12.110

[12] Mende M., Schwarz D., Steinbach C., Boldt R., Schwarz S.: Simultaneous adsorption of heavy metal ions and anions from aqueous solutions on chitosan - Investigated by spectrophotometry and SEM-EDX analysis. Colloids and Surfaces A: Physicochemical and Engineering Aspects, 510, 275-282 (2016). https://doi.org/10.1016/j.colsurfa.2016.08.033

[13] Li A., Lin R., Lin C., He B., Zheng T., Lu L., Cao Y.: An environment-friendly and multi-functional absorbent from chitosan for organic pollutants and heavy metal ion. Carbohydrate Polymers, 148, 272-280 (2016). https://doi.org/10.1016/j.carbpol.2016.04.070

[14] Sorlier P., Viton C., Domard A.: Relation between solution properties and degree of acetylation of chitosan: Role of aging. Biomacromolecules, 3, 1336-1342 (2002). https://doi.org/10.1021/bm0256146

[15] Kandile N. G., Mohamed H. M., Mohamed M. I.: New heterocycle modified chitosan adsorbent for metal ions (II) removal from aqueous systems. International Journal of Biological Macromolecules, 72, 110-116 (2015). https://doi.org/10.1016/j.ijbiomac.2014.07.042

[16] Salehi E., Daraei P., Shamsabadi A. A.: A review on chitosan-based adsorptive membranes. Carbohydrate Polymers, 152, 419-432 (2016).

https://doi.org/10.1016/j.carbpol.2016.07.033 
[17] Borgohain R., Boruah P. K., Baruah S.: Heavy-metal ion sensor using chitosan capped $\mathrm{ZnS}$ quantum dots. Sensors and Actuators B: Chemical, 226, 534-539 (2016). https://doi.org/10.1016/j.snb.2015.11.118

[18] Esmaeili A., Khoshnevisan N.: Optimization of process parameters for removal of heavy metals by biomass of $\mathrm{Cu}$ and Co-doped alginate-coated chitosan nanoparticles. Bioresource Technology, 218, 650-658 (2016). https://doi.org/10.1016/j.biortech.2016.07.005

[19] Zhang L., Zeng Y., Cheng Z.: Removal of heavy metal ions using chitosan and modified chitosan: A review. Journal of Molecular Liquids, 214, 175-191 (2016). https://doi.org/10.1016/j.molliq.2015.12.013

[20] Ferhat M., Kadouche S., Drouiche N., Messaoudi K., Messaoudi B., Lounici H.: Competitive adsorption of toxic metals on bentonite and use of chitosan as flocculent coagulant to speed up the settling of generated clay suspensions. Chemosphere, 165, 87-93 (2016). https://doi.org/10.1016/j.chemosphere.2016.08.125
[21] Qiu H., Liang C., Yu J., Zhang Q., Song M., Chen F.: Preferable phosphate sequestration by nano-La(III) (hydr)oxides modified wheat straw with excellent properties in regeneration. Chemical Engineering Journal, 315, 345-354 (2017). https://doi.org/10.1016/j.cej.2017.01.043

[22] Zhang Q., Teng J., Zou G., Peng Q., Du Q., Jiao T., Xiang $\mathrm{J} .:$ Efficient phosphate sequestration for water purification by unique sandwich-like MXene/magnetic iron oxide nanocomposites. Nanoscale, 8, 7085-7093 (2016). https://doi.org/10.1039/c5nr09303a

[23] Zhang Q., Du Q., Hua M., Jiao T., Gao F., Pan B.: Sorption enhancement of lead ions from water by surface charged polystyrene-supported nano-zirconium oxide composites. Environmental Science and Technology, 47, 6536-6544 (2013). https://doi.org/10.1021/es400919t 